\title{
A fast specular reflection removal based on pixels properties method
}

\author{
Shahrizan Jamaludin ${ }^{1}$, Nasharuddin Zainal $^{2}$, W. Mimi Diyana W. Zaki ${ }^{3}$ \\ ${ }^{1}$ Department of Maritime Technology, Faculty of Ocean Engineering Technology and Informatics, \\ Universiti Malaysia Terengganu, Malaysia \\ ${ }^{2,3}$ Department of Electrical, Electronic and Systems Engineering, Faculty of Engineering and Built Environment, \\ Universiti Kebangsaan Malaysia, Malaysia
}

\begin{tabular}{l}
\hline \hline Article Info \\
\hline Article history: \\
Received Feb 2, 2019 \\
Revised Apr 8, 2020 \\
Accepted May 14, 2020
\end{tabular}

\section{Keywords:}

Human iris Iris recognition Iris segmentation Pixels properties Specular reflection

\begin{abstract}
Iris recognition has been around for many years due to an extensive research on the uniqueness of human iris. It is well known that the iris is not similar to each other which means every human in the planet has their own iris pattern and cannot be shared. One of the main issues in iris recognition is iris segmentation. One element that can reduce the accuracy of iris segmentation is the presence of specular reflection. Another issue is the speed of specular reflection removal since the iris recognition system needs to process a lot of irises. In this paper, a specular reflection removal method was proposed to achieve a fast and accurate specular reflection removal. Some modifications were implemented on the existing pixels properties method. Based on the results, the proposed method achieved the fastest execution time, the highest segmentation accuracy and the highest SSIM compared to the other methods. This proves that the proposed method is fast and accurate to be implemented in the iris recognition systems.
\end{abstract}

This is an open access article under the CC BY-SA license.

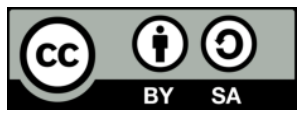

\section{Corresponding Author:}

Shahrizan Jamaludin,

Department of Maritime Technology,

Faculty of Ocean Engineering Technology and Informatics,

Universiti Malaysia, Malaysia.

Email: shahrizanj@umt.edu.my

\section{INTRODUCTION}

Iris recognition is one of many biometric systems [1] that use human body for human identification. The examples of biometric systems are DNA, face, ear, palmprint, fingerprint and many more. The iris recognition system uses human iris as an object or token for human identification and verification. The iris is located in the eye, between the sclera (limbic boundary) and pupil (pupillary boundary). Sclera is the white part of the eye, while pupil is the black circle at the middle of the eye. Human eye is also surrounded by eyelids [2] and eyelashes in order to protect the eye from the dust and particle that can cause interference to the eye. According to the researches, the iris has a high recognition accuracy [3, 4]. The iris recognition is easy to use [5] since it can be captured using camera and video. The iris pattern located in the iris is very stable [6] and does not change during our lifetime [7]. The real-time applications of iris recognition system can be seen in the UK immigration system, the US police departments, the passport presentation of Schiphol airport (Netherlands), the UAE border security system and residency permit applications system, the Iraq's control points of entry into Fallujah and the Pakistan-Afghanistan border of repatriation centre [8].

The advantage of iris compared to the other biometrics such as it has rich features like crypts, collarettes, coronas, freckles, rings and arching ligaments which makes it unique with variety [9]. The iris 
also has rich pattern and texture such as ridges, pigment spots and furrows [10]. The longitudinal and radial muscles formed the structure and texture of iris [11]. Each iris has a high degree of freedom with its own features [12]. The probability of two same irises is only 1 in $10^{72}$ [13]. Even the pattern of the right and left irises are totally different [7]. Other than that, the iris recognition has high security [14] due to the location of iris itself, protected by the eyelids. It also is so reliable [15] since it cannot be easily lost and forgotten such as key, token and password.

There are five main stages in the iris recognition system. The first stage is iris acquisition where the human iris is captured using camera or video. The captured image can be a stand still image or a moving image. Moreover, the iris can be captured under the near infra-red [16] or visible wavelength [17]. The second stage is iris segmentation. Iris segmentation is crucial in order to isolate the iris from the other eye elements such as the sclera, pupil, eyelid and eyelash. The pupillary and limbic boundary will be detected in order to isolate the iris. The third stage is normalization where the isolated iris is converted from the circular shape into a rectangular polar coordinate. This stage can compensate the changing iris pattern due to the erosion of pupil [18]. The fourth stage is feature extraction. In this stage, the iris from the rectangular coordinate is extracted in order to capture its pattern. Then, the iris pattern is encoded into a binary code which is called as an iris code. Finallly, the iris code is compared with the iris code in the database for identification and verification.

There are many methods can be used for iris recognition. The most popular methods are Hough transform [19] and Integro-differential operator [3]. Both methods are considered as the pioneer of iris recognition. Other than that, the method using only partial part of iris [18] and LoG filters [20] can also be used for iris segmentation. The iris recognition system itself can be implemented in personal computer [4], FPGA [21] and GPU [22].

The previous specular reflection removal methods used many different ways to eliminate or reduce specular reflection. The specular reflection itself can obstruct the rich iris pattern, which can reduce the accuracy of iris segmentation. Other than that, the iris recognition system needs to process a lot of human irises. Thus, the specular reflection removal must be fast to process a lot of irises. Moreover, the existing specular reflection removal methods are time consuming thus not ideal to be implemented in the real-time system. Due to that, this paper proposed a specular reflection removal method based on the pixels properties in order to tackle above problems. An improvement and modification of the existing pixels properties method is conducted to achieve a fast and accurate specular reflection removal.

\section{PREVIOUS WORKS}

Specular reflection normally happens during image acquisition either the subject is stand still or moving by using camera and video. This phenomenon is common because of the source of lighting that might interfere during the acquisition of image. The source of lighting comes from the sunlight, camera flash, reflection and any object that can produce light. In iris recognition, the specular reflection can be observed on the surface of iris, sclera and pupil. Specular reflection might also occur on the pupillary boundary and limbic boundary. According to [23], specular reflection can reduce the quality of iris pattern. Moreover, it can contribute to the false and poor iris localization [14]. This happens because of the iris localization depends on the location of pupil. In the researches in [3, 17-19], the center of pupil is used as a base to segment the accurate iris region.

Previously, the specular reflection is eliminated with a simple method as in [17]. The authors proposed an image complementation technique twice and a holes filling technique. According to the authors, the proposed specular reflection and iris segmentation methods can improve the speed of iris segmentation algorithm. Meanwhile in [24], the morphological closing is proposed for the specular reflection removal which can reduce the reflection itself. Then in [25], the bilinear interpolation is used for the removal of specular reflection. This method used the adaptive threshold in order to reduce reflection in the affected area. After that in [26], an extension of linear interpolation is used. According to the authors, this technique is much better than the bilinear interpolation. Other than that, the morphological operator can be used to fill holes in the affected area of iris [27].

The top hat [28] and bottom hat [14] filters are also used but with mixed results. According to [14], all methods proposed as above can be used to eliminate and reduce specular reflection. Unfortunately, those methods required more time to execute which can slow the process of iris recognition. The methods that required more time to execute are the bottom hat filter [14] and bilinear interpolation [25]. Due to that, the authors in [14] proposed a new method which is based on the pixels properties, which is much faster than the other methods. However, the pixels properties method has many operations to eliminate the specular reflection. Due to that, this paper will modify and optimize the pixels properties method in order to further reduce the execution time to eliminate specular reflection. 


\section{RESEARCH METHOD}

It is important to ensure the pupil region is free from the specular reflection since a lot of works [3, 17-19] used the center of pupil as a base for iris segmentation. Specular reflection can be spotted in a shape of circle which is similar to the shape of pupil. Due to that, the iris segmentation algorithm might falsely detect the specular reflection as the pupil region, which can contribute to the false iris segmentation.

In this work, the pixels properties methods as in $[14,18]$ are used as a reference for the proposed specular reflection removal method. The pixels properties method is a technique that calculates the pixels and properties of the selected area. In [14], the pixels properties method is used along with the unsharp masking, morphological closing and flood-fill methods. Firstly, the iris image quality is improved with the unsharp masking. The unsharp masking is implemented to enhance the iris image contrast for easier detection of specular reflection. Moreover, this technique can sharpen the texture of iris image. The second step is to apply a threshold value for the pupil region. All regions in the iris image will be analyzed to detect the potential location of specular reflection, based on the given threshold value. In this case, the threshold value is assigned to 40 . Then, the detected regions are analyzed by using the pixels properties method in order to determine the correct specular reflection. The third step is to apply the morphological closing to enlarge, then shrink the detected specular reflection. Finally, the specular reflection is eliminated using the flood-fill method.

Meanwhile in [18], the threshold value is obtained from the pretest of 100 iris images. The average value of pupil region from the pretest is assigned as a threshold value, which is 35 . After that, the pixels properties method is used to detect the specular reflection. Then, the morphological closing and flood-fill methods are used to eliminate specular reflection. However, both methods required many operations which are three and four methods respectively to remove the specular reflection. Logically, the less operations to remove specular reflection can lead to a faster execution time.

Due to that, both methods can be optimized by reducing the number of methods or operations to remove the specular reflection, thus can achieve a faster execution time. Moreover, method [14] used a threshold value of 40 to detect the specular reflection. This value is not suitable and accurate especially when dealing with a lot of iris images. The average value should be obtained instead of one fixed value as in [18]. Other than that, the unsharp masking might be useful to enhance the quality of iris pattern. However, this method is not really necessary to remove the specular reflection. Moreover, both morphological closing and flood-fill methods can be combined due to their similar characteristic or function which is to fill holes of the detected specular reflection. Due to above factors, a modified specular reflection removal method is proposed.

Firstly, the threshold value is obtained from the average value of 100 iris images of CASIA v4 database [29]. According to [14], the value below 40 is suitable as a threshold value due to the characteristic of pupil region which is one of the largest and darkest elements in the iris images. The average value of 34 is obtained, thus this value is assigned as a threshold value. Next, the pixels properties method is applied based on the assigned threshold value. The pixels properties method manages to locate many possible specular reflections in the iris image that corresponds with the threshold value. After that, the individual properties of each possible specular reflections are analyzed in order to locate the exact pupil region. This region is obtained from the largest area of each possible specular reflection. This is because the pupil region has a solid texture and one of the largest elements in the iris image, along with the eyelashes. However, due to the threshold value, the eyelashes are not detected as a pupil region. The pixels properties method contains the information of pixels list and matrix location of all possible specular reflections. Pixels list is the number of all possible specular reflections, and matrix location stores the coordinates of each possible specular reflections. From this information, the pixels properties method manages to identify the exact pupil region. As the exact region has been located, the specular reflections in the pupil region can be eliminated by using the flood-fill method. The parameters of flood-fill method are set where four-connected element is used for two separate backgrounds with a radius of 15 pixels. The flood-fill method will use these parameters to close any holes with a radius of 15 pixels. The value of 15 pixels is obtained due to the standard radius value of the specular reflection as stated in $[14,18]$. The same intensity of surrounded pixels will fill the dark region, which is surrounded by the light region in the pupil region. The artefacts and unconnected regional minima will also be removed by the flood-fill method. Finally, zero value is assigned in order to enhance the contrast of pupil region that is free from specular reflection. The proposed method can be observed in Figure 1. For the performance evaluation of segmentation accuracy, [1] obtained from [30] is used where,

$$
S A_{k}=\frac{1}{r \times c} \sum_{i=1}^{r} \sum_{j=1}^{c}\left(I_{i, j} \otimes G_{i, j}\right)
$$


$G_{i, j}$ is the ground-truth iris image, $I_{i, j}$ is the segmented iris image, $i$ and $j$ are the pixel coordinates, $r$ and $c$ are the height and width of iris image, and $\otimes$ is the Boolean XOR operation. Then, [2] by [30] is used to obtain the overall segmentation accuracy, $S A$ by calculating the average of iris images, $N$ where,

$$
S A=\frac{1}{N} \sum_{k=1}^{N} S A_{k}
$$

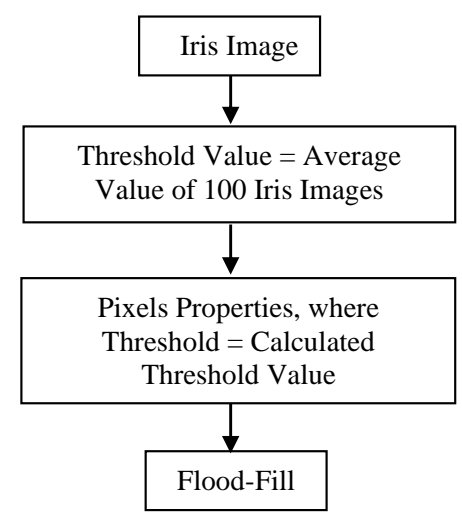

Figure 1. The proposed method

For the performance evaluation of image quality, the structural similarity index (SSIM) is used as in [14]. The SSIM is a method used to measure the similarity of two iris images where it will estimate the image degradation from the perceive changes. The quality of iris images is analyzed in order to determine whether the iris pattern is changed during the operation of specular reflection removal. The unchanged iris pattern will record a SSIM value near to 1. In (3) shows the SSIM equation.

$$
\operatorname{SSIM}=\frac{\left(2 \mu_{x} \mu_{y}+c_{1}\right)\left(2 \sigma_{x y}+c_{2}\right)}{\left(\mu_{x}^{2}+\mu_{y}^{2}+c_{1}\right)\left(\sigma_{x}^{2}+\sigma_{y}^{2}+c_{2}\right)}
$$

\section{RESULTS AND DISCUSSION}

The proposed specular reflection removal method is developed on Windows 8.1, Intel Core i5 2.3 GHz and $4 \mathrm{~GB}$ RAM workstation with MATLAB software. The 400 iris images taken from the CASIA v4 iris database is used in this experimented. The experimental result can be observed in Table 1. Based on the result, the proposed method achieved the fastest execution time compared to the methods in $[14,18]$ respectively. This happened because of the proposed method had less operations and methods, in order to remove the specular reflection. Method [14] used the unsharp masking and morphological closing for the specular reflection removal. Meanwhile in [18], only morphological closing was used. In the other hand, the proposed method did not use both unsharp masking and morphological closing in its specular reflection removal algorithm which managed to reduce the execution time.

Table 1. The performance of execution time and segmentation accuracy

\begin{tabular}{cccc}
\hline Methods & Average Execution Time (s) & Segmentation Accuracy (\%) & SSIM \\
\hline$[14]$ & 0.19 & 94 & 0.9872 \\
{$[18]$} & 0.17 & 95 & 0.9889 \\
Proposed method & 0.12 & 97 & 0.9921 \\
\hline
\end{tabular}

Moreover, method in [18] achieved a faster execution time than [14] due to less methods implemented for the specular reflection removal. Method [18] used the morphological closing, while did not use the unsharp masking compared to the method in [14]. Additionally, the unsharp masking method needed to duplicate the original iris image, in order to subtract the original iris image from the unsharp iris image, thus required more time to execute. Meanwhile in morphological closing, three operations were used 
to perform this method. First, the foreground boundaries were enlarged, then the background boundaries were shrunk, and finally the holes were eliminated. The morphological closing also used the same parameter as the flood-fill method which was redundant and required more sources.

Next, the proposed method was compared in terms of segmentation accuracy. According to the result, the proposed method managed to achieve the highest segmentation accuracy than the methods in $[14,18]$. This happened because of the proposed method can segment the correct pupil and iris regions compared to the methods in $[14,18]$. The proposed method can remove the specular reflection a little bit better than the methods of $[14,18]$. A high segmentation accuracy can contribute to a high recognition accuracy for the verification and identification of iris recognition system. However, the segmentation accuracy of both methods was still high and acceptable to be implemented in the iris recognition system.

In terms of image quality, the proposed method achieved the highest quality than the methods in $[14,18]$. This happened due to the highest SSIM value compared to the other methods. The proposed method managed to remove the specular reflection without much affecting the quality of iris pattern in the iris image. As the result showed, all three methods achieved an acceptable SSIM value. However, the proposed method was much better to be used in the iris recognition system because of the less effect on the iris pattern. For conclusion, the proposed specular reflection removal method achieved the fastest execution time, the highest segmentation accuracy and the highest SSIM value compared to the methods in $[14,18]$. This proved that the proposed method is fast and accurate to be used in the offline and real-time iris segmentation systems. The specular reflection removal results can be observed in Figure 2.

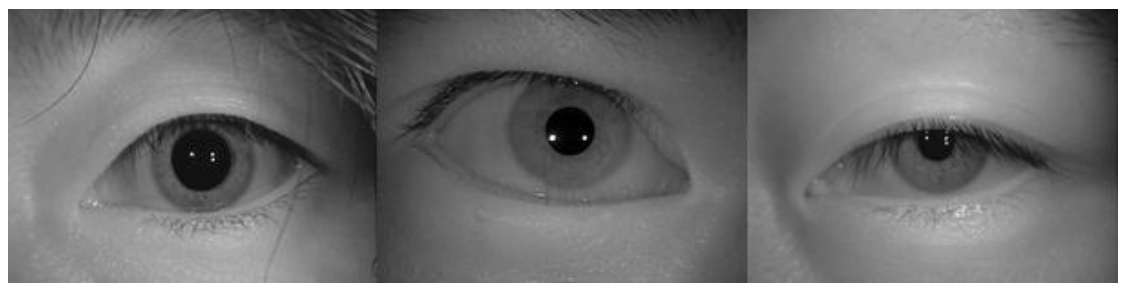

(a)

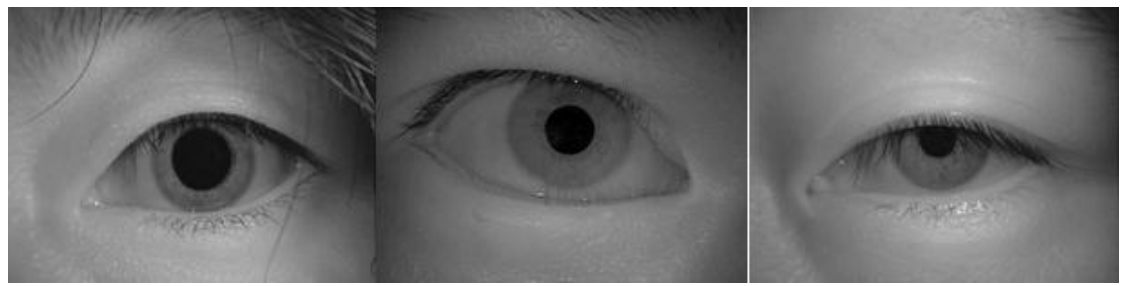

(b)

Figure 2. The specular reflection removal results, (a) The original iris image, (b) After removal

\section{CONCLUSION}

In this study, the specular reflection removal method based on the pixels properties was proposed. The previous pixels properties method was modified in order to obtain the better performance to remove the specular reflection. The proposed method excluded a few operations and methods in order to optimize the specular reflection removal method. In terms of execution time, the proposed method managed to achieve the fastest execution time because of it used less operations. The proposed method did not use both unsharp masking and morphological closing in its algorithm which managed to reduce the execution time. In terms of segmentation accuracy, the proposed method achieved the highest accuracy than the referred methods due to the proposed method can segment the correct specular reflection, pupil and iris regions. In terms of image quality, the proposed method recorded the highest SSIM compared to the other methods. The specular reflection was removed without affecting the iris pattern quality in the iris image. These results showed that the proposed method is fast and accurate to be used in the offline and real-time iris recognition systems. For future work, the proposed method can be implemented in the iris recognition system in order to analyze the performance of recognition accuracy. 


\section{REFERENCES}

[1] Khandait S. P., Thool R. C., Khandait P. D., "Hybrid Facial Geometry Algorithm for Facial Feature Extraction and Expression Recognition using ANFIS and BPNN," Bulletin of Electrical Engineering and Informatics, vol. 2, no. 1, pp. 11-22, March 2013.

[2] Nayak R. K., Agarwal S., "Comparision of Iris Identification by using Modified SIFT and SURF Keypoint Descriptor," Ph.D. dissertation, Electronics and Instrumentation Engineering, National Institute of Technology Rourkela, Rourkela, Odisha, India, 2013.

[3] J. G. Daugman, "High confidence visual recognition of persons by a test of statistical independence," in IEEE Transactions on Pattern Analysis and Machine Intelligence, vol. 15, no. 11, pp. 1148-1161, Nov 1993.

[4] S. Jamaludin, N. Zainal, W. M. D. W. Zaki, "Iris Recognition Based on the Modified Chan-Vese Active Contour," Jurnal Teknologi, vol. 78, no. 10-13, pp. 13-17, 2016.

[5] Kadry S, Smaili K, "A Design and Implementation of a Wireless Iris Recognition Attendance Management System," Information Technology and Control, vol. 36, no. 3, pp. 323-329, 2015.

[6] M. M. Khedkar and S. A. Ladhake, "Robust human iris pattern recognition system using neural network approach," International Conference on Information Communication and Embedded Systems ICICES, Chennai, pp. 78-83, 2013.

[7] John Daugman and Cathryn Downing, "Epigenetic Randomness Complexity and Singularity of Human Iris Patterns," Proceedings of the Royal Society B: Biological Sciences, vol. 268, pp. 1737-1740, Aug 2001.

[8] Daugman J., "Evolving Methods in Iris Recognition and Implications from 200 Billion Iris Comparison," IEEE International Conference on Biometrics: Theory, Applications, and Systems, (BTAS07), 1993, (online). http://www. cse. nd. edu/BTAS_07/John_Daugman_BTAS.pdf.

[9] A Muron, J. Pospisil, "The Human Iris Structure and Its Usages," Acta Universitatis Palackianae Phisica, vol. 39, pp. 87-95, 2000.

[10] J. Wayman, "Book review: Handbook of Iris Recognition," in IET Biometrics, vol. 3, no. 1, pp. 41-43, March 2014.

[11] Y. D. Khan, F. Ahmad, M. W. Anwar, "A Neuro-Cognitive Approach for Iris Recognition using Back Propagation," World Applied Sciences Journal, vol. 16, no. 5, pp. 678-685, 2012.

[12] Dae Sik Jeong, et al, "A New Iris Segmentation Method for Non-Ideal Iris Images," Image and Vision Computing, vol. 28, no. 2, pp. 245-260, Feb 2010.

[13] L. Flom, A. Safir, "Iris recognition system," U.S. Patent No. 4,641,349. 3 Feb. 1987.

[14] Shahrizan Jamaludin, Nasharuddin Zainal, W. Mimi Diyana W. Zaki, "The Removal of Specular Reflection in Noisy Iris Image,” Journal of Telecommunication, Electronic and Computer Engineering, vol. 8, no. 4, pp. 59-64, 2016.

[15] Raja Abdullah Raja Ahmad, Muhammad Imran Ahmad, Mohd Nazrin Md Isa, Said Amirul Anwar, "Face Recognition Using Assemble of Low Frequency of DCT Features," Bulletin of Electrical Engineering and Informatics, vol. 8, no. 2, pp. 541-550, June 2019.

[16] Maulisa Oktiana, Fitri Arnia, Yuwaldi Away, Khairul Munadi, "Features for Cross Spectral Image Matching: A Survey," Bulletin of Electrical Engineering and Informatics, vol. 7, no. 4, pp. 552-560, Dec 2018.

[17] A. Radman, K. Jumari and N. Zainal, "Fast and reliable iris segmentation algorithm," in IET Image Processing, vol. 7, no. 1, pp. 42-49, Feb 2013.

[18] Shahrizan Jamaludin, Nasharuddin Zainal \& W Mimi Diyana W Zaki, "Sub-Iris Technique for Non-Ideal Iris Recognition," Arabian Journal for Science and Engineering, vol. 43, no. 12, pp. 7219-7228, 2018.

[19] R. P. Wildes, "Iris recognition: an emerging biometric technology," in Proceedings of the IEEE, vol. 85, no. 9, pp. 1348-1363, Sept. 1997.

[20] R. M. Abbas, T. M. Khan, M. A. U. Khan and H. A. Khan, "Fast and accurate iris segmentation methods using LoG filters," 2019 International Conference on Computing, Electronics \& Communications Engineering iCCECE, London, United Kingdom, pp. 124-129, 2019.

[21] Tariq M. Khan, Donald G. Bailey, Mohammad A. U. Khan \& Yinan Kong, "Efficient Hardware Implementation Strategy for Local Normalization of Fingerprint Images," Journal of Real-Time Image Processing, vol. 16, pp. 1263-1275, 2019.

[22] Shahrizan Jamaludin, Nasharuddin Zainal and W. Mimi Diyana W. Zaki, "GPU Implementation of Sub-Iris Technique in Iris Recognition System,” Pertanika Journal of Science and Technology, vol. 25, pp. 263-274, 2017.

[23] Kwang Yong Shin, et. al, "New Iris Recognition Method for Noisy Iris Images," Pattern Recognition Letters, vol. 33, no. 8, pp. 991-999, 2012.

[24] Brian O'Connor, Kaushik Roy, Joseph Shelton, and Gerry Dozier, "Iris Recognition Using Fuzzy Level Set and GEFE," International Journal of Machine Learning and Computing, vol. 4, no. 3, pp. 225-231, 2014.

[25] Z. He, T. Tan, Z. Sun and X. Qiu, "Toward Accurate and Fast Iris Segmentation for Iris Biometrics," in IEEE Transactions on Pattern Analysis and Machine Intelligence, vol. 31, no. 9, pp. 1670-1684, Sep 2009.

[26] Walid Aydi, Nouri Masmoudi, Lotfi Kamoun, "New Corneal Reflection Removal Method Used in Iris Recognition System," World Academy of Science, Engineering and Technology, vol. 5, no. 5, pp. 898-902, 2011.

[27] Amit Bendale, Aditya Nigam, Surya Prakash, Phalguni Gupta, "Iris Segmentation Using Improved Hough Transform," Emerging Intelligent Computing Technology and Applications, ICIC 2012. Communications in Computer and Information Science, Springer, Berlin, Heidelberg, pp. 408-415, 2012.

[28] C. Tsai, H. Lin, J. Taur and C. Tao, "Iris Recognition Using Possibilistic Fuzzy Matching on Local Features," in IEEE Transactions on Systems, Man, and Cybernetics, Part B Cybernetics, vol. 42, no. 1, pp. 150-162, Feb. 2012.

[29] Tieniu Tan, Zhaofeng He, Zhenan Sun, "Efficient and Robust Segmentation of Noisy Iris Images for NonCooperative Iris Recognition," Image and Vision Computing, vol. 28, no. 2, pp. 223-230, Feb 2010.

[30] H. Proenca and L. A. Alexandre, "The NICE.I: Noisy Iris Challenge Evaluation - Part I," 2007 First IEEE International Conference on Biometrics: Theory, Applications, and Systems, Crystal City, VA, pp. 1-4, 2007. 